\title{
Research for Application of Mobile Digital Library
}

\author{
Zhang Xin $^{\mathrm{a}}{ }^{*}$, Song Dingli ${ }^{\mathrm{b}}$, Yu Fuxing ${ }^{\mathrm{c}}$ and Yan Shu ${ }^{\mathrm{d}}$ \\ North China University of Science and Technology \\ asdlhr1006@sohu.com, bsdlhr617@sohu.com, cyfx626@126.com, d1183526779@qq.com
}

\begin{abstract}
Keywords: WAP (Wireless Application Protocol); mobile digital library; application; system implementation
\end{abstract}

Abstract. With the rapid development of China's science and technology, information technology has been used widely, especially in the construction of mobile digital library. We usually read with books in the past, each campus will build the library, to facilitate student learning and searching information. But with the use of mobile digital library, students can consult through its information, they should not dedicated to the library. It's save a lot of time for students and short cut to cumbersome procedures, and students can get the required information promptly. In this way, library materials not only allow students to master, but also convenient for all people. Through the analysis for mobile digital library applications status quo and characteristics of WAP2.0 technology, this thesis establish a functional structure model of mobile digital library and system-building programs, and took the analyzed data as a basis to achieve mobile digital library system in reality.

\section{Analysis for the current situation of mobile digital library at home and abroad}

Library construction is an integral part of the school also the paradise for students to learn and search information. In order to further convenient student access to information, reduce search, find the middle of the cumbersome procedures, it is urgent to optimize the library and innovate network service model information of new library. Therefore, the use of mobile information and communication technology for the construction of the library community technology platform to become the inevitable trend of domestic and international library development ${ }^{[1]}$. With the application of information technology to library construction, the formation of mobile digital library system, praised by the readers ${ }^{[2]}$.

Research on mobile digital library at home and abroad. Because our country is a developing country, and compared to Western Europe and other developed countries, China's comparative weakness in the mobile communication and information technologies, unlike Western Europe and other countries, through analysis, research, document, presented, in the following gives several more classic mode for wireless network services ${ }^{[3]}$ :

Study with a SMS way. This is a short message service for search for book, through mobile phone short message to accept, publishing for readers to provide SMS alerts and advisory services. For example: If, as the date to extend the borrowed books, simply edit out cell phone short message "01+ barcode books" do not have to go to the library to handle; To query whether the desired collection of books in the house, just edit short interest "02+ title" you can know. A typical case is in Finland, the Finnish National Library opening the SMS service, convenient to know information about the books. SMS opened the main function of renewals, expiration reminders, appointment books notice, failure to retrieve feedback information, advice, readers, reading tips weekly, etc., the reader is free to use ${ }^{[4]}$.

Study with a i-Mode service way. This technique is a mobile phone Internet service of wireless communication network be implemented in Japan a that can combine mobile information technology and library to enable the students to use. Users can log in Tokyo University Library website with via mobile phone, discover book store information about the library's, and taking online reading, while also can urging the user, renewals and other services.

Study with a WAP site service way. WAP is a mobile terminal to provide Internet content and advanced value-added services unified global open protocol standards, which is a simple protocol for wireless networks. American University of South Alabama Library is a "roofless Library Project" 
Using PDA retrieve library resources, the reader can be connected wirelessly via the mobile communication network of the library, the library online catalog of resources Search.

Study with a J2ME services manner. This is a highly optimized mode of java runtime environment, mainly for consumer electronics devices, it has take java language and platform-independent characteristic migrate to small electronic devices, and between the mobile wireless device can be programmed to share. You can also design library system into a mobile phone handset client software, readers can be this client downloaded to the phone, access to wireless mobile services provided by the library software library ${ }^{[5]}$.

Research on the status of domestic mobile library. As China's mobile digital library rather late, research bookmobile application is still in its infancy, far behind the developed Western countries ${ }^{[6]}$. China's mobile library has many defects, such as: service system is imperfect, service capacity is weak, not enough thorough study of various service models, application of mobile information technology is not mature enough, but still managed to get some results. Later, with the rapid development of WAP technology, the mobile library applications are more mature, with a representative of mobile digital library are:

First, when it comes to our capital Beijing, Peking University library system began tentative SMS platform to use. It can through SMS platform, using a mobile phone number to register, develop appropriate custom library service, the system can automatically periodically send the appropriate message service, users no matter where they are notified library books due reminder, appointment reminders and expiration reminders also reminded other services.

Shanghai is the first case of opening the country to run a mobile phone readers personalized service. Users can access the site via mobile phone, go to the website in the "My Account" access to their own circumstances Jie Du, conduct phone books Renewal Services. Now the library implementation of "one card pass", the reader can be queried via the card information and loan information, and overdue reminder service, to modify and other personal information.

The "Pocket National Library" platform which pushed by the National Library's mobile WAP site, including mobile digital library, short message service, the National Library WAP sites, mobile phones reading and others.

\section{Studying for System Construction Design}

The analysis of system construction objectives. Design of mobile digital library is based on WAP2.0. WAP2.0 technology, mobile communication technology, mobile services into the digital library system ${ }^{[7]}$. Now people are basically phones, users only need Internet access through mobile GPRS or the Internet in other ways, you can at any time in the library holdings information check out the. Related resource information including: a variety of dynamic query in the library, information library of published queries of reader guidelines, information inquiry and library book recommendations, reference service, borrow books on behalf of, loan repayment service and other information services.

Research on the overall system architecture design. Mobile digital library is used by B / S mode, this model, as long as registered users can in a different time and place, connected to the library service system via the user interface requirements. At the same time such a system development, maintenance, upgrade management it is very easy to manage personnel and administrative costs needed to be very low. Mobile digital library system uses a classic hierarchical MVC design ideas, the whole system is divided into three layers: A. Model layer, business logic. This layer is the main part of the mobile digital library, its main function is responsible for the achievement of specific business logic, and state management capabilities. B. The view layer, user layer ${ }^{[8]}$. The application layer is the system user part is mainly responsible for the connection with the user, to provide users with the appropriate information library; by accepting user input data, and then transferred to query the service status, a request to store the database, but not any substantive business processes, but is responsible for connecting to convert data request signal. C. The controller layer, data access layer. This layer is the 
role of the entire system control all business processes, will work with the model layer, view layer to achieve; it is primarily responsible for the interpretation of an object from the input view, these views data into the system can handle, while identifying users request, be converted into model-specific call; at the same time, it is also able to handle the results of the event and model logic from the execution of the model, and finally call the appropriate view feedback to the user.

Research on the system function architecture design. Based on the functional structure of the mobile digital library system WAP2.0, mainly before, backstage management system consisting of two modules. Front desk management system is the user interface system, is responsible for establishing a connection with the user window, the library will convey relevant information to the user, for example: library news announcement, the latest developments in the library, library book recommendations, one-stop service and, finally, convenient service platform; one of the one-stop service platform is the core module of the system, the system is mainly responsible for the majority of business processing library. The main role of background management system is related to the information content of the user interface will be updated in time.

\section{The analysis of the implementation of the system}

Network systems mobile digital library research. WAP2.0-based mobile digital library is in the existing library network services platform as the basis for implementation, and in which the use of WAP2.0 wireless network technology research. Construction of the system's program consists of three parts: a.WAP terminal part, is what we call the Library ordinary viewer and library membership; b.WAP gateways, mainly responsible for the entire library network, information technology-related businesses to provide and maintain mobile communications companies and the like ISP; c.WAP content server, which is a mobile digital library WAP content server.

Development of mobile digital library system operating environment for analysis. Development and implementation of the system is very important, about the normal operation of mobile digital library. Its development environment and runtime environment must be chosen carefully, reasonable and scientific arrangement, to find suitable development runtime environment. Because the system development time will involve issues WAP2.0 wireless network protocols and TCP / IP network protocol, etc., it is necessary to consider the system hardware birth to different places need to use different network interfaces. In previous work, usually using an analog form to test the performance of the entire system, until after the completion of simulation test, can determine when the actual implementation, we can use mobile phones and other mobile devices by actual users, go to the mobile digital library the reality of the system test. System development environment to build tools: QuickWAP2.0; system simulation testing tools: Win-wap4.0 browser.

Research on mobile digital library system database design. After create a database using SQL Sever2005 database management system, accordingly data operation: connect to the database operation or operations are commonly used code range (insert records, delete records, update records) in Table 1. 


\begin{tabular}{cc}
\hline Data tables & Description \\
\hline adn in & Account information of user \\
adn in list & Access records of administrator \\
City Data & Information of city \\
City Item & City Type \\
Find Work & Service Guide Information \\
Guest Book & Reader Service \\
Hot Ent & Information Collection Electronic \\
News class & Resources \\
News Data & Extended to remind the reader type \\
Product List & Overdue reminders \\
Site Announce & Information of new books \\
Site New & News Information \\
Visit Count & News information \\
Table l Data tables and & Access records of client \\
\hline
\end{tabular}

Table 1 Data tables and corresponding function description

Research on production in detail. Through designed to the mobile digital library system, after complete all design work. The overall function of the mobile digital library has been tested and passed the test, it can properly put into use. For the mobile digital library development in our country into examples prove, but also played a catalytic role to a certain extent.

After the completion of the system, we also need to develop appropriate management practices, and build a harmonious order. Due to its special multi-service object is now the University Library, the reader users uneven quality, the need to develop appropriate standards, to guide users to actively participate. The first is to specify a user's behavior, without abuse and other uncivilized behavior; when the quiz, you need to use standard Mandarin to communicate with the library system, otherwise it will not recognize the language, resulting in business for failure; the development of appropriate incentives and punitive measures. Use rating system of rewards, the implementation of accumulated points system in the user's personal information, the implementation of a substantial reward at each level to specified points, to encourage readers to participate in the mobile digital library user system. Users can ask their own learning through this platform issues that arise from using the platform sharing to resolve, and the question and answer storage. The most important thing is to provide our users to comply with the order, the use of the library system platform to effectively solve their own problems, to play its due role mobile digital library.

\section{Conclusion}

According to the current actual situation, based on WAP2.0 mobile digital library system has been completed, and put into practical applications. Users can WAP2.0 browser mobile terminals, anytime, anywhere into the library, you can easily, quickly and freely consult books from libraries to information and services can be handled. Throughout the commissioning of the project, the actual working order throughout the mobile digital library system, both in the data collection, transmission, storage, aspects of publication, or in user traffic handling, etc., are reflected in the actual nature of the system , applicability, economy, safety and reliability, is now the preferred Library Innovation development. As China is in the primary stage of exploration, so the system in the development process will inevitably be inadequate, in the coming days, scientists still need to innovate, improve and perfect the entire mobile digital library system to achieve the best effect.

\section{References}

[1] Jiang Lifu. Witkey and library information consulting services. Tu Shu Guan Gong Zuo Yu Yan Jiu. 2008(2): 54-56. 
[2] Xu Yibo, Zhong Shaochun. Mobile learning system based on WEB. Journal of Jilin University(Information Science Edition). 2009,27(3):278-282.

[3] Jia Hong. The research on personalized service of digital library. Modern Information,2006(3): 71-73.

[4] He Wei, Cao Jindan, Diao Yunmei. The application of mobile equipment in libraries' patrons service. Information Science, 2006(5):767-771.

[5] Ouyang Feng. Web data mining and academic digital library personalized service. Modern Information, 2008(1):103-104.

[6] Liu Xia. The application of mobile terinals in the library. Sci-tech Information Development \& Economy,2008(12):11-13.

[7] Huang Weiyu. The research on personalized service of digital library. Modern Information,2007(10): 81-83.

[8] Sun Ping. Research of library service technology based on mobile terminal. Modern Information,2007(12): 133-135. 